TRANSACTIONS OF THE

AMERICAN MATHEMATICAL SOCIETY

Volume 361, Number 8, August 2009, Pages 4233-4245

S 0002-9947(09)04783-7

Article electronically published on February 10, 2009

\title{
A $\Pi_{1}^{1}$-UNIFORMIZATION PRINCIPLE FOR REALS
}

\author{
C. T. CHONG AND LIANG YU
}

\begin{abstract}
We introduce a $\Pi_{1}^{1}$-uniformization principle and establish its equivalence with the set-theoretic hypothesis $\left(\omega_{1}\right)^{L}=\omega_{1}$. This principle is then applied to derive the equivalence, to suitable set-theoretic hypotheses, of the existence of $\Pi_{1}^{1}$-maximal chains and thin maximal antichains in the Turing degrees. We also use the $\Pi_{1}^{1}$-uniformization principle to study Martin's conjectures on cones of Turing degrees, and show that under $V=L$ the conjectures fail for uniformly degree invariant $\Pi_{1}^{1}$ functions.
\end{abstract}

\section{INTRODUCTION}

We identify a real $x$ with a subset of $\omega$. A real is $\Pi_{1}^{1}$ if and only if it is definable or equivalent, over second order arithmetic, to a formula that begins with a universal set or function quantifier followed by an arithmetic relation. There is a notion of $\Pi_{1}^{1}$-inductive definitions for reals. Kleene's $\mathcal{O}$ is perhaps the penultimate example of such an object.

One may introduce a parallel notion of inductive definition for sets of reals. If $Q: 2^{2^{\omega}} \rightarrow 2^{2^{\omega}}$ is a function, with the additional property that $Q(\emptyset) \subseteq 2^{\omega}$, then it is progressive (Sacks [15) if $Q(X) \supseteq X$ for all $X \subseteq 2^{\omega}$. $Q$ is monotonic if $Q(X) \subseteq$ $Q(Y)$ whenever $X \subseteq Y$. Given an ordinal $\beta$, let $\bar{Q}^{0}=Q(\emptyset), Q^{\beta+1}=Q\left(Q^{\beta}\right) \cup Q^{\bar{\beta}}$, and, for a limit ordinal $\lambda>0, Q^{\lambda}=\bigcup_{\beta<\lambda} Q^{\beta}$. $\Pi_{1}^{1}$-monotonic relations have been studied by various authors, including Spector [18, Aczel and Richter [1] as well as Cenzer [4. The main interest was in identifying the "ordinal of $Q$ ", i.e. the least ordinal $\beta$ where $Q^{\beta+1}=Q^{\beta}$. However, the study of specific inductively defined relations has seen less activity.

The Kondo-Addison Uniformization Theorem states that given a $\Pi_{1}^{1}$ relation $\mathrm{P}(x, y)$, there is a $\Pi_{1}^{1}$ relation $\mathrm{P}^{*}$ such that for all $x$, if there is a $y$ satisfying $\mathrm{P}(x, y)$, then there is a unique $y$ satisfying $\mathrm{P}^{*}(x, y)$. Conversely, for any $x, y$, if $\mathrm{P}^{*}(x, y)$, then $\mathrm{P}(x, y)$. Thus $\mathrm{P}^{*}$ is a $\Pi_{1}^{1}$ function that uniformizes $\mathrm{P}$. There are $\Pi_{1}^{1}$ progressive relations with very useful properties, which may be applied to derive $\Pi_{1}^{1}$-uniformization principles. Such principles may in turn be used to investigate interesting problems in descriptive set theory and recursion theory. In this paper we

Received by the editors August 14, 2007.

2000 Mathematics Subject Classification. Primary 03D28, 03E35, 28A20.

The research of the first author was supported in part by NUS grant WBS 146-000-054-123.

The second author was supported by NSF of China Grant No. 10701041, Research Fund for Doctoral Programs of Higher Education, No. 20070284043, and Scientific Research Foundation for Returned Overseas Chinese Scholars. 
present an example to illustrate this point. More specifically, we introduce an inductively defined $\Pi_{1}^{1}$-uniformization principle for a $\Pi_{1}^{1}$ relation on the hyperdegrees of reals, and show some of its applications.

There is a class of problems on the existence of definable maximal collections or families of reals with prescribed properties that are of interest to set theorists and recursion theorists. A naive approach, under $V=L$, is to proceed by induction over $L_{\omega_{1}}$, where in a $\Sigma_{1}\left(L_{\omega_{1}}\right)$ fashion a definable maximal family of reals is constructed. Such a family would typically yield a $\Sigma_{2}^{1}$ set of reals over second order arithmetic. Much harder work is required if one aims for a collection of lower complexity. Refining the naive construction, Kunen and Miller 13 have obtained a $\Pi_{1}^{1}$-maximal family of almost disjoint sets. In [5] and [6], we investigated the problem of the existence of $\Pi_{1}^{1}$-maximal chains and thin (i.e., without a perfect subset) antichains in the Turing degrees, and proved the equivalence of the existence of these sets with respective set-theoretic hypotheses on $\omega_{1}$ and $2^{\omega}$. Underlying the constructions were similar techniques and intuitions that pointed to a general principle applicable to different situations, perhaps at a deeper level. Our investigation leads to the formulation of a $\Pi_{1}^{1}$-uniformization principle, denoted $\mathfrak{I}$, discussed below. The principle is applied to establish the key results in [5] and [6], and also to study Martin's conjectures on Turing degrees. It is worth noting that the idea of coding reals in the formulation of the principle $\mathfrak{I}$ was also used in the work of Kunen and Miller to construct a $\Pi_{1}^{1}$-maximal family of almost disjoint sets.

Let $\mathcal{C}$ be the set of reals $x$ such that $x \in L_{\omega_{1}^{x}}$, where as usual $\omega_{1}^{x}$ is the least admissible ordinal not recursive in $x$. It is well known that $\mathcal{C}$ is $\Pi_{1}^{1}$ and that every $\Pi_{1}^{1}$ set of reals contains an element of $\mathcal{C}$ (Sacks [15]). Elements of $\mathcal{C}$ enjoy special properties that are not available to those in its complement. For example, if $x$ is a master code in the sense of Jensen's fine structure theory [10] (or equivalently an "arithmetical copy" in the sense of Boolos and Putnam [3]), then $x \in \mathcal{C}$. In [5] and [6], the $\Pi_{1}^{1}$-maximal chain and thin antichain were constructed exploiting this fact, so that each element of these sets was chosen to be in $\mathcal{C}$ with specific properties, including requiring it to have double Turing jump, that is, Turing equivalent to a master code. However, the double jump, while sufficient for the constructions of the sets in [5] and [6], is probably too restrictive and not necessary for a deeper understanding of the " $\Pi_{1}^{1}$ phenomenon", and the use of fine structure theory as well as Borel determinacy, while interesting and revealing, might also be avoided when viewed in a different light. This is the intuition behind our search for a general principle. The motivation is that the principle should not only prove theorems obtained in [5] and [6], but also be useful for studying other problems. The test problem we consider is Martin's conjectures on cones of Turing degrees for degree invariant functions. Since this is known to be false under $A C$, and the conjectures are posed under the assumption of the Axiom of Determinacy $A D$, and known to hold for uniformly degree-invariant functions assuming $A D$ (Steel [19, Slaman and Steel [16]), we focus our attention on uniformly definable degree-invariant functions - in this case $\Pi_{1}^{1}$ - to learn how far $A C$ and the conjectures may co-exist in a model of set theory without full $A D$.

The organization of the paper is as follows. In $\S 2$ we recall some standard notions, definitions and results in hyperarithmetic theory, highlighting in particular the Spector-Gandy Theorem which characterizes $\Pi_{1}^{1}$ sets of reals. In $\S 3$ we introduce a $\Pi_{1}^{1}$-progressive relation and use it to define the $\Pi_{1}^{1}$-uniformization principle $\mathfrak{I}$. We 
show the validity of this principle under the set-theoretic hypothesis $\left(\omega_{1}\right)^{L}=\omega_{1}$, and demonstrate that $\mathfrak{I}$ and the hypothesis are equivalent. In $\S 4$ we show that the existence of a $\Pi_{1}^{1}$-maximal chain and a thin $\Pi_{1}^{1}$-maximal antichain is a consequence of, and equivalent to, $\mathfrak{I}$ under appropriate set-theoretic axioms. In $\S 5$ we apply the uniformization principle to study Martin's conjectures, and show that it fails with $\Pi_{1}^{1}$-degree-invariant functions under $V=L$. In particular, we exhibit $\Pi_{1}^{1}$ uniformly degree-invariant functions $f$ on the reals such that $f(x) \equiv_{\mathrm{T}} x$ on cofinally (in the sense of Turing reducibility) many $x$ 's but $f(x)$ is not Turing equivalent to $x$ on any cone of Turing degrees. Our final result states that $<_{M}$, the partial ordering defined on degree-invariant functions (see $\S 5$ ), is not a pre-well-ordering on $\Pi_{1}^{1}$ uniformly degree-invariant functions. Thus both parts of Martin's conjectures do not hold for uniformly degree-invariant functions definable at the $\Pi_{1}^{1}$ level of the analytic hierarchy.

\section{Preliminaries}

We adopt set-theoretic notation throughout this paper. In particular, small Roman letters $x, y, z, \ldots$ refer to reals, while Greek letters $\alpha, \beta, \ldots$ denote ordinals. The collection of paths of a perfect tree $T$ is denoted $[T]$, and \langle\rangle is a recursive bijection from $\omega \times \omega$ to $\omega$. Finally, $<_{L}$ denotes the $\Delta_{2}^{1}$ well-ordering of the reals in Gödel's constructible universe $L$.

The reader is assumed to be familiar with hyperarithmetic theory as presented in Sacks [15], which is used as the standard reference in this paper. Here we recall some notions that may be considered to be at the intersection of set theory and recursion theory and which are needed throughout the paper.

For each real $x, \omega_{1}^{x}$ denotes the least ordinal which is not an order type of an $x$-recursive well-ordering of $\omega$ (the least $x$-admissible ordinal). Kleene constructed a $\Pi_{1}^{1}(x)$ complete set $\mathcal{O}^{x}$ with a $\Pi_{1}^{1}(x)$ well-founded relation $<_{\mathcal{O}^{x}}$ on $\mathcal{O}^{x}$. $\mathcal{O}^{x}$ is the hyperjump of $x$ and if $x \in L$, then $\mathcal{O}^{x} \in \mathcal{C}$, where $\mathcal{C}$ is the set defined in $\S 1$, and $\omega_{1}^{\mathcal{O}^{x}}$ is the least admissible ordinal after $\omega_{1}^{x}$. The height of the ordering $<_{\mathcal{O}^{x}}$ on $\mathcal{O}^{x}$ is exactly $\omega_{1}^{x}$ (see [15]). Furthermore, Kleene's construction of $\mathcal{O}^{x}$ is uniform. In other words, the relation $\left\{\left(x, \mathcal{O}^{x}\right) \mid x \in 2^{\omega}\right\}$ is $\Pi_{1}^{1}$. A fact that will be used implicitly is that given reals $x$ and $y, x$ is hyperarithmetic in $y$ (written $x \leq_{h} y$ ) if and only if $x$ is $\Delta_{1}^{1}$ in $y$, and this is in turn equivalent to $x \in L_{\omega_{1}^{y}}[y]$.

A result of central importance to this paper, due to Spector and Gandy, is an application of Kleene's theory in the characterization of $\Pi_{1}^{1}$ sets of reals. This states that $A \subseteq 2^{\omega}$ is $\Pi_{1}^{1}$ if and only if there is an arithmetical relation $\mathrm{R}(x, y)$ such that

$$
x \in A \Leftrightarrow \exists y \leq_{h} x(\mathrm{R}(x, y)) .
$$

Boolos and Putnam [3] also studied the relationship between Kleene's theory and Gödel's constructible universe $L$. They proved that hyperarithmetic reals are exactly the reals in $L_{\omega_{1}^{\mathrm{CK}}}$. Moreover $\omega_{1}^{x}$ is the least ordinal $\alpha>\omega$ such that $L_{\alpha}[x]$ is admissible (see [2] and [15]). By their result, the Spector-Gandy Theorem may be restated as follows:

Theorem 2.1 (Spector, Gandy). A set $A$ of reals is $\Pi_{1}^{1}$ if and only if there is a $\Sigma_{0}$-formula $\varphi$ (in the language of $Z F$ set theory) such that

$$
y \in A \Leftrightarrow\left(\exists x \in L_{\omega_{1}^{y}}[y]\right)\left(L_{\omega_{1}^{y}}[y] \models \varphi(x, y)\right) .
$$


Recall that $\mathcal{C}=\left\{x \mid x \in L_{\omega_{1}^{x}}\right\}$. Every nonempty $\Pi_{1}^{1}$ set contains an element in $\mathcal{C}$ (15. III. Lemma 9.3). A relativized version of this result, whose proof is a straightforward adaptation of the original proof, is the following (we will denote in the rest of the paper $\omega^{(x, y)}$ for $\left.\omega_{1}^{x \oplus y}\right)$ :

Proposition 2.2. If $A \neq \emptyset$ is $\Pi_{1}^{1}(x)$ for some real $x$, then there is a $y \in A$ such that $y \in L_{\omega_{1}^{(y, x)}}[x]$.

A particularly useful characterization of $\Pi_{1}^{1}$ sets of reals is due to Mansfield and Solovay ([15] III. Theorem 9.5, Mansfield [11, Solovay [17]):

Theorem 2.3 (Mansfield and Solovay). Let $A$ be a $\Pi_{1}^{1}$ set of reals. The following are equivalent:

(i) A contains a constructibly coded perfect subset;

(ii) there is an $x \in A$ such that $x \notin \mathcal{C}$.

\section{A $\Pi_{1}^{1}$-UNIFORMIZATION PRINCIPLE}

Definition 3.1. A binary relation $\mathrm{P}(x, y)$ on $2^{\omega} \times 2^{\omega}$ is cofinally progressive if for every real $x$, the set $\{y \mid \mathrm{P}(x, y)\}$ is cofinal in the hyperdegrees, i.e. for each $z$, there is a real $y \geq_{h} z$ such that $\mathrm{P}(x, y)$ holds.

Lemma 3.2. Assume $\left(2^{\omega}\right)^{L}=2^{\omega}$. If a $\Pi_{1}^{1}$ relation $\mathrm{P}$ is cofinally progressive, then for every real $x$ and $z$ there is a real $y \geq_{h} z$ such that $y \in \mathcal{C}$ and $\mathrm{P}(x, y)$.

Proof. Suppose $\left(2^{\omega}\right)^{L}=2^{\omega}$ and $\mathrm{P}$ is cofinally progressive. Then for any reals $x, z$, the set $A=\left\{y \mid x \oplus z \in L_{\omega_{1}^{y}} \wedge \mathrm{P}(x, y)\right\}$ is a nonempty $\Pi_{1}^{1}(x \oplus z)$ set. Thus, by Proposition 2.2, there is a real $y \in A$ for which $y \in L_{\omega_{1}^{(x, z, y)}}[x \oplus z]$. Since $x \oplus z \in L_{\omega_{1}^{y}}$, we have $x \oplus z \leq_{h} y$ and so $\omega_{1}^{(x, z, y)}=\omega_{1}^{y}$. Then $y \in L_{\omega_{1}^{(x, z, y)}}[x \oplus z]=L_{\omega_{1}^{y}}$ and $\mathrm{P}(x, y)$.

Given a countable set $A$ and a real $x$, we say that $x$ codes $A$ if $\left\{(x)_{n} \mid n \in \omega\right\}=A$ where $(x)_{n}=\{m \mid m \in \omega \wedge\langle n, m\rangle \in x\}$.

A cofinally progressive relation generates a progressive relation (in the sense defined in $\S 1)$ as follows: Let $\mathrm{P}(x, y)$ be cofinally progressive. For each $X \subseteq 2^{\omega}$, let $Q(X)=X \cup\{y \mid \exists x \in X \mathrm{P}(x, y)\}$. Then it is immediate that $Q(X) \supseteq X$ for all $X$. However, an operation such as $Q$ may be too general to be useful and this motivates a refinement of $Q$ to something close to a uniformization function for well-defined sets. Now if $\mathrm{P}(x, y)$ is $\Pi_{1}^{1}$, the Kondo-Addison Theorem guarantees a uniformization function for $\mathrm{P}$. However, our interest is in strong uniformization, i.e. a function that uniformizes "initial segments of reals" that does roughly the following: If $x$ codes a set of reals in $\mathcal{C}$ such that any two elements $w<_{L} z$ in $x$ satisfy $\mathrm{P}(w, z)$, then there is a "least" $y \in \mathcal{C}$ such that $x \leq y$ and $\mathrm{P}(x, y)$ holds. This is made precise by the $\Pi_{1}^{1}$-uniformization principle $\mathfrak{I}$ :

$\Pi_{1}^{1}$-uniformization principle $\mathfrak{I}$. If a binary relation $\mathrm{P}(x, y)$ is $\Pi_{1}^{1}$ and cofinally progressive, then there is a $\Pi_{1}^{1}$ set $A \subseteq \mathcal{C}$ such that:

(i) $\left|\leq_{L}\right| A \mid$, the height of $\leq_{L}$ on $A$, is $\omega_{1}$;

(ii) $\forall y\left(y \in A \Longrightarrow \exists x\left(x \in L_{\omega_{1}^{y}} \wedge x\right.\right.$ codes the set $\left.\left.\left\{z \mid z \in A \wedge z<_{L} y\right\} \wedge \mathrm{P}(x, y)\right)\right)$.

Theorem 3.3. $V=L$ implies $\mathfrak{I}$. 
Proof. Suppose $V=L$, and $\mathrm{P}$ is $\Pi_{1}^{1}$ and cofinally progressive. Note that for each constructibly countable $\beta$, there is an $\alpha>\beta$ such that $\left(L_{\alpha+1} \backslash L_{\alpha}\right) \cap 2^{\omega} \neq \emptyset$. For a given ordinal $\alpha$ and $X \subseteq \alpha \times \omega$, denote by $X[\beta]$ the real $\{n \in \omega \mid(\beta, n) \in X\}$. We may regard $X$ as a sequence of reals of length $\alpha$.

Since $\mathrm{P}$ is $\Pi_{1}^{1}$, by the Spector-Gandy Theorem there is a $\Sigma_{0}$ formula $\psi(x, y, s)$ such that

$$
\mathrm{P}(x, y) \Leftrightarrow L_{\omega_{1}^{(x, y)}}[x, y] \models \exists s \psi(x, y, s) .
$$

Assuming $V=L$, we define a partial function $F$, which we will show to be total on $\omega_{1} \times \bigcup_{\alpha<\omega_{1}} 2^{\alpha \times \omega}$, as follows:

For each $\alpha<\omega_{1}$ and set $X \subseteq \alpha \times \omega$ with $\alpha<\omega_{1}$, we define $F(\alpha, X)$ to be the real $z$ such that $L_{\omega_{1}^{(X, z)}}[X, z]$ satisfies the following properties:

(1) There is a $\beta \geq \alpha$ so that $z \in L_{\beta+1} \backslash L_{\beta}$.

(2) There is a real $x \in L_{\omega_{1}^{(X, z)}} \operatorname{coding} X$.

(3) There is a limit ordinal $\gamma$ and an element $s \in L_{\gamma}$ so that $L_{\gamma}[X, z] \models$ $\psi(x, z, s)$.

(4) If $(t, y, \mu)<_{L}(s, z, \gamma)$, then $L_{\mu}[X, y] \models \psi(x, y, t) \Longrightarrow \omega_{1}^{(X, y)}<\beta$.

(5) If $(t, y, \mu)<_{L}(s, z, \gamma)$ and $L_{\mu}[X, y] \models \psi(x, y, t)$, then either no real $r \in$ $L_{\omega_{1}^{(X, y)}} \operatorname{codes} X$ or $y \notin L_{\omega_{1}^{(X, y)}}$.

Since $L_{\omega_{1}^{(X, z)}}[X, z]=L_{\omega_{1}^{(X, z)}},<_{L}$ is uniformly $\Delta_{1}$ in $L_{\omega_{1}^{(X, z)}}[X, z]$. Hence $(1)-(5)$ are uniformly $\Sigma_{1}$ in $L_{\omega_{1}^{(X, z)}}[X, z]$ (see [8]).

We show that $F(\alpha, X)$ is defined for each $\alpha<\omega_{1}$ if $X$ is countable.

Fix $(\alpha, X)$. Since $V=L$, there is a countable $\gamma^{\prime}>\alpha$ with a real $x \in L_{\gamma^{\prime}}$ coding $X$. Since $V=L$ and $\mathrm{P}$ is cofinally progressive, by Lemma 3.2, there is a $\beta>\gamma^{\prime}$ so that $\left(L_{\beta+1} \backslash L_{\beta}\right) \cap 2^{\omega} \neq \emptyset$ and a real $z \in L_{\beta+1} \backslash L_{\beta}$ so that $x \oplus z \in$ $L_{\omega_{1}^{z}}$ and $L_{\gamma} \models \psi(x, z, s)$ for some limit ordinal $\gamma<\omega_{1}^{z}$ and $s \in L_{\gamma}$. Note that $L_{\omega_{1}^{z}}=L_{\omega_{1}^{(X, z)}}[X, z]$ and $L_{\gamma}=L_{\gamma}[X, z]$ for all sufficiently large $\gamma<\omega_{1}^{z}$. So (1)(3) are satisfied. Obviously we can assume that $(s, z, \gamma)$ is the $<_{L}$-least satisfying these properties. So (4)-(5) are satisfied. By the absoluteness of $<_{L}$, one concludes that $F$ is a well-defined function defined on each $(\alpha, X)$ where $\alpha<\omega_{1}$ and $X$ is countable.

Observe that (1)-(4) are $\Sigma_{1}$ statements. By (4), one verifies that (5) is a $\Delta_{1}$ statement. Hence there is a $\Sigma_{0}$ formula $\varphi(\alpha, X, z, s)$ such that $F(\alpha, X)=z$ if and only if $L_{\omega_{1}^{(X, z)}}[X, z] \models(\exists s) \varphi(\alpha, X, z, s)$.

Thus we may perform transfinite induction on $\alpha$ to define a sequence $\left\{z_{\alpha} \mid \alpha<\omega_{1}\right\}$ using $F$. However, the resulting sequence in general yields a set of reals that is $\Sigma_{1}$ over $L_{\omega_{1}}$, i.e. $\Sigma_{2}^{1}$ but not necessarily $\Pi_{1}^{1}$ over second order arithmetic. To ensure that $\Pi_{1}^{1}$-ness is achieved, we refine the approach as follows.

Define $G(\alpha)=z$ if and only if $\alpha<\omega_{1}^{z}$ and there is a function $f: \alpha+1 \rightarrow 2^{\omega}$ with $f \in L_{\omega_{1}^{z}}[z]$ so that for all $\beta \leq \alpha, f(\beta)=F(\beta,\{(\gamma, n) \mid n \in f(\gamma) \wedge \gamma<\beta\})$ and $f(\alpha)=z$. Since $L_{\omega_{1}^{z}}[z]$ is admissible, $\{f(\gamma) \mid \gamma \leq \alpha\} \in L_{\omega_{1}^{z}}[z]$. So $G(\alpha)=z$ if and only if there is a function $f: \alpha+1 \rightarrow 2^{\omega}$ with $f \in L_{\omega_{1}^{z}}[z]$ such that

$$
L_{\omega_{1}^{z}}[z] \models((\forall \beta \leq \alpha)(\exists s) \varphi(\beta,\{(\gamma, n) \mid n \in f(\gamma) \wedge \gamma<\beta\}, s, f(\beta))) \wedge f(\alpha)=z .
$$


Since $L_{\omega_{1}^{z}}[z]$ is admissible, $G$ is $\Sigma_{1}$-definable. In other words, $G(\alpha)=z$ if and only if there is a function $f: \alpha+1 \rightarrow 2^{\omega}$ with $f \in L_{\omega_{1}^{z}}[z]$ such that

$L_{\omega_{1}^{z}}[z] \models((\exists t)(\forall \beta \leq \alpha)(\exists s \in t) \varphi(\beta,\{(\gamma, n) \mid n \in f(\gamma) \wedge \gamma<\beta\}, s, f(\beta))) \wedge f(\alpha)=z$.

Define the range of $G$ to be $A$. Then $z \in A$ if and only if there exists an ordinal $\alpha<\omega_{1}^{y}$ and a function $f: \alpha+1 \rightarrow 2^{\omega}$ with $f \in L_{\omega_{1}^{z}}[z]$ such that

$L_{\omega_{1}^{z}}[z] \models((\exists t)(\forall \beta \leq \alpha)(\exists s \in t) \varphi(\beta,\{(\gamma, n) \mid n \in f(\gamma) \wedge \gamma<\beta\}, s, f(\beta))) \wedge f(\alpha)=z$.

So $A$ is $\Pi_{1}^{1}$.

All that remains is to show that $G$ is a well-defined total function on $\omega_{1}$. This can be done using the same argument as that for showing the recursion theorem over admissible structures (see Chapter I, Theorem 6.4 of Barwise 2]). The only nontrivial part is to argue that the function $f$ defined above exists. This can be done by induction. Let $\alpha<\omega_{1}$ be given and suppose that $\left\{f_{\beta}\right\}_{\beta<\alpha}$ is a uniformly $\Sigma_{1}$-increasing (i.e. $f_{\beta} \supset f_{\gamma}$ for $\beta>\gamma$ ) sequence such that each $f_{\beta}$ is an $f$ at stage $\beta<\alpha$, as required. Let $f_{\alpha}$ be such that $f_{\alpha} \in L_{\omega_{1}^{z}}[z]$ and for all $\beta \leq \alpha$, $f_{\alpha}(\beta)=F\left(\beta,\left\{(\gamma, n) \mid n \in f_{\beta}(\gamma) \wedge \gamma<\beta\right\}\right)$ and $f_{\alpha}(\alpha)=z$, where $F(\alpha, X)=z$ and $X=\left\{(\gamma, n) \mid \exists \beta\left[\gamma \leq \beta<\alpha \& n \in f_{\beta}(\gamma)\right]\right\}$. Note that $X=\{(\gamma, n) \mid \gamma \leq$ $\left.\left.\alpha \& n \in f_{\gamma}(\gamma)\right]\right\}$ by the absoluteness. Then $f_{\alpha} \supset f_{\beta}$ for all $\beta<\alpha$ and satisfies the requirement of such an $f$ at stage $\alpha$.

By (1) and (2) above, $(x, z) \in L_{\gamma}$ and $L_{\gamma} \in L_{\omega_{1}^{z}}$. So $A \subset \mathcal{C}$.

Since $\mathrm{P}$ is cofinally progressive, (i) in $\mathfrak{I}$ is true.

For (ii): If $y \in A$, then $y=G(\alpha)$ for some $\alpha<\omega_{1}$. By the definition of $G$, there is a real $x \in L_{\omega_{1}^{G(\alpha)}}$ such that $x$ codes the set $\{G(\beta) \mid \beta<\alpha\}$ and $\mathrm{P}(x, G(\alpha))$ holds. But obviously $\beta<\alpha$ if and only if $G(\beta)<_{L} G(\alpha)$. Hence (ii) holds.

It turns out that the $\Pi_{1}^{1}$-uniformization principle $\mathfrak{I}$ is equivalent to the settheoretic assumption $\left(\omega_{1}\right)^{L}=\omega_{1}$, as we now show.

Theorem 3.4. $\left(\omega_{1}\right)^{L}=\omega_{1}$ if and only if the $\Pi_{1}^{1}$-uniformization principle $\mathfrak{I}$ is true.

Proof. The "if" direction is immediate: Choose $\mathrm{P}(x, y)$ to express $x \leq_{\mathrm{T}} y(x$ is Turing reducible to $y$ ). Then $\mathrm{P}$ is cofinally progressive. Let $A$ be a $\Pi_{1}^{1}$ set satisfying $\mathfrak{I}$ for $\mathrm{P}$, of $<_{L}$-height $\omega_{1}$. Thus $\left(\omega_{1}\right)^{L}=\omega_{1}$.

For the other direction, suppose $\mathrm{P}$ is $\Pi_{1}^{1}$ and cofinally progressive. Then for any pair of reals $x, z \in L$, the set $U_{x, z}=\left\{y \mid y \geq_{h} z \wedge \mathrm{P}(x, y)\right\}$ is a nonempty $\Pi_{1}^{1}(x, z)$ set. So there must be some $y \in U_{x, z} \cap L$. Then by the absoluteness of $\Pi_{1}^{1}$ relations,

$$
L \models \mathrm{P} \text { is cofinally progressive. }
$$

By Theorem 3.3 , there is a $\Pi_{1}^{1}$ set $A$ so that $(A)^{L}$ witnesses the correctness of the uniformization principle in $L$.

Since $\left(\omega_{1}\right)^{L}=\omega_{1}$, (i) in the uniformization principle is true in $V$.

Since the statement " $x \in L_{\omega_{1}^{x}}$ " is $\Pi_{1}^{1}$ and

$$
L \models \forall x\left(x \in A \Longrightarrow x \in L_{\omega_{1}^{x}}\right),
$$

by the absoluteness of $\Pi_{2}^{1}$ statements, $V \models \forall x\left(x \in A \Longrightarrow x \in L_{\omega_{1}^{x}}\right)$. Hence $A \subset \mathcal{C}$.

Choose any $y \in A$. Since $A \subseteq L, y \in L$. Then there exists a real $x \in L_{\omega_{1}^{y}}$ so that

$$
L \models x \text { codes the set }\left\{z \mid z \in A \wedge z<_{L} y\right\} \wedge \mathrm{P}(x, y) .
$$


Since $A \subset L, x$ codes the set $\left\{z \mid z \in A \wedge z<_{L} y\right\}$. Since the relation $\mathrm{P}$ is $\Pi_{1}^{1}$ and $x, y \in L$, by the absoluteness of $\Pi_{1}^{1}$ relations, $\mathrm{P}(x, y)$ holds. Hence (ii) follows.

Thus the $\Pi_{1}^{1}$-uniformization principle $\mathfrak{I}$ holds.

One may relativize the $\Pi_{1}^{1}$-uniformization principle to admit real parameters to obtain the boldface version of $\mathfrak{I}$. Then Theorem 3.4 may be generalized to state: The boldface $\boldsymbol{\Pi}_{1}^{1}$-uniformization principle $\mathfrak{I}$ fails if and only if there is no real $x$ so that $\left(\omega_{1}\right)^{L[x]}=\omega_{1}$. This leads to the following result:

Corollary 3.5. The statement " $Z F C+\boldsymbol{\Pi}_{1}^{1}$-uniformization principle $\mathfrak{I}$ is false" is consistent if and only if " $Z F C+$ there exists an inaccessible cardinal" is consistent.

Proof. If $\mathfrak{I}$ fails, then $\left(\omega_{1}\right)^{L[x]}<\omega_{1}$ for all $x$, so that the latter is inaccessible in $L$. Conversely, suppose $Z F C+$ "there is an inaccessible cardinal" is consistent. Then, by Levy collapse, $Z F C+$ " $\omega_{1}$ is inaccessible in $L$ " is consistent. Now in a model say $\mathcal{M}$ with such a property, $\omega_{1}>\left(\omega_{1}\right)^{L[x]}$ for all $x$. So the $\boldsymbol{\Pi}_{1}^{1}$-uniformization principle fails in $\mathcal{M}$.

\section{Maximal Chains AND THIN MAXimal ANTICHAins IN THE TURING DEGREES}

In this section, we apply the uniformization principle $\mathfrak{I}$ to solve the existence problem of $\Pi_{1}^{1}$-maximal chains and thin $\Pi_{1}^{1}$-maximal antichains in the Turing degrees. These problems were studied in [5] and [6] using direct constructions involving the Boolos-Putnam analysis of constructible reals. Here we derive the results from the principle $\mathfrak{I}$.

Theorem 4.1 (Chong and Yu [5]). Assume $\left(\omega_{1}\right)^{L}=\omega_{1}$. There is a $\Pi_{1}^{1}$-maximal chain in the Turing degrees.

Proof. Define a binary relation $\mathrm{P}$ as follows:

$$
\mathrm{P}(x, y) \Leftrightarrow y \text { is a minimal cover of }\left\{(x)_{n} \mid n \in \omega\right\} .
$$

It was shown in [5] that $\mathrm{P}$ is a $\Delta_{1}^{1}$ cofinally progressive relation (in the language of the current paper). Hence by Theorem 3.4 there is a $\Pi_{1}^{1}$ set $A$ as prescribed. $\mathfrak{I}($ ii) implies that $A$ is a maximal chain.

In the case of a maximal antichain, a diagonal argument shows that under $Z F C$ there is a thin maximal antichain in the Turing degrees. Hence the definability of a thin set with such a property becomes particularly interesting. Since every set of reals whose degrees form a maximal antichain has size $2^{\aleph_{0}}$, it cannot be $\boldsymbol{\Sigma}_{1}^{1}$ (else it would contain a perfect subset). The next level of definability is then $\Pi_{1}^{1}$. We apply Theorem 3.4 to construct a thin $\Pi_{1}^{1}$-maximal antichain in the Turing degrees.

Theorem 4.2. Assume $\left(2^{\omega}\right)^{L}=2^{\omega}$. There exists a $\Pi_{1}^{1}$ thin maximal antichain in the Turing degrees.

Proof. Define a binary relation $\mathrm{P}(x, y)$ as follows:

(1) $\left\{(x)_{n} \mid n \in \omega\right\}$ is not an antichain; or

(2) $\left\{(x)_{n} \mid n \in \omega\right\} \cup\{y\}$ is an antichain and (2a) $x \oplus(y)_{0} \in L_{\omega_{1}^{y}}$;

\footnotetext{
${ }^{1}$ To rule out trivial cases: if there exist $m \neq n$ such that $(x)_{m}=(x)_{n}$, then we assume $(x)_{m}$ and $(x)_{n}$ are the same set.
} 
(2b) $\left\{(x)_{n} \mid n \in \omega\right\} \cup\left\{(y)_{0}\right\}$ is an antichain;

(2c) for every $z<_{L}(y)_{0},\left\{(x)_{n} \mid n \in \omega\right\} \cup\{z\}$ is not an antichain.

As shown in 6, $\mathrm{P}$ is a cofinally progressive relation. Note that $(2 \mathrm{c})$ is equivalent to

$$
L_{\omega_{1}^{(x, y)}}[x, y] \models \exists \beta\left(y_{0} \in L_{\beta} \wedge \forall z \in L_{\beta}\left(z<_{L} y \Longrightarrow \exists n\left((x)_{n} \geq_{\mathrm{T}} y \vee y \geq_{\mathrm{T}}(x)_{n}\right)\right)\right) .
$$

Thus $\mathrm{P}$ is $\Pi_{1}^{1}$. Hence by Theorem 3.4 there is a $\Pi_{1}^{1}$ set $A$ with the prescribed properties. Using $\mathfrak{I}(\mathrm{ii})$, we show by induction on $<_{L}$ that $A$ is an antichain.

Suppose $x \in A$ and $\left\{y \mid y \in A \wedge y<_{L} x\right\}$ is an antichain. By $\Im($ ii), there is a $z$ coding $\left\{y \mid y \in A \wedge y<_{L} x\right\}$ so that $\mathrm{P}(z, y)$ holds. Then by $(2),\left\{(z)_{n} \mid n \in \omega\right\} \cup\{y\}$ is an antichain. So $A$ is an antichain. Note that $A \subset \mathcal{C}$. This together with Theorem 2.3 implies that $A$ is a thin set. By (2b) above, $A$ is maximal.

Note that despite the use of a uniformization principle, the proofs of the above theorems still appeal to metamathematical assumptions to establish the results. One may wonder if this approach is at all avoidable. It turns out that this is a necessary route:

Theorem 4.3 (ZFC). $\quad$ (i) There is a thin $\Pi_{1}^{1}$-maximal antichain of Turing degrees if and only if $\left(2^{\omega}\right)^{L}=2^{\omega}$.

(ii) There is a thin $\Pi_{1}^{1}$ (boldface) maximal antichain of Turing degrees if and only if $\left(2^{\omega}\right)^{L[x]}=2^{\omega}$ for some real $x$.

Proof. (i): Suppose $A$ is a thin $\Pi_{1}^{1}$-maximal antichain. Then by Theorem 2.3 , $A \subset L$. Now let $x$ be a real. By a theorem of Cooper [7, there is a real $y$ of minimal degree such that $x \leq_{T} y^{\prime}$. Since $A$ is a maximal antichain, there is a real $z \in A$ with $z \geq_{\mathrm{T}} y$. So $x \leq_{\mathrm{T}} z^{\prime}$. Hence $x \in L$.

Conversely, suppose $\left(2^{\omega}\right)^{L}=2^{\omega}$. Fix a $\Pi_{1}^{1}$ set $G$ as in Lemma 4.2. Since the statement " $G$ is an antichain in the Turing degrees" is $\Pi_{2}^{1}$ and

$$
L \models \text { " } G \text { is an antichain in the Turing degrees", }
$$

$G$ is an antichain in the Turing degrees in $V$ by absoluteness. Fix a real $x$. Since $\left(2^{\omega}\right)^{L}=2^{\omega}, x \in L$. The statement $T(x)$ : "there exists $y \in G$ so that $y$ is Turing comparable with $x "$ is $\Sigma_{2}^{1}(x)$ and $L \models T(x)$. It follows that $T(x)$ is true. Thus $G$ is a maximal antichain.

(ii): Relativize the proof of (i).

A direct proof of Theorem 4.3 may be found in [6]. It follows that to construct a model in which there is no thin $\boldsymbol{\Pi}_{1}^{1}$-maximal antichain of Turing degrees, one only needs to refute $\mathrm{CH}$ in the model.

Theorem 3.4 explains why an additional hypothesis is required to show Theorem 4.2, even with help from the uniformization principle $\mathfrak{I}$, since the assumption $\left(\omega_{1}\right)^{L}=\omega_{1}$ is not sufficient to construct a $\Pi_{1}^{1}$-thin maximal antichain. Nevertheless, the following theorem still holds:

Proposition 4.4. If $\left(\omega_{1}\right)^{L}=\omega_{1}$, then there is a $\Pi_{1}^{1}$-thin antichain of size $\aleph_{1}$ in the Turing degrees. 
Proof. Define a binary relation $\mathrm{P}(x, y)$ :

(1) $\left\{(x)_{n} \mid n \in \omega\right\}$ is not an antichain, or

(2) $\left\{(x)_{n} \mid n \in \omega\right\} \cup\{y\}$ is an antichain.

Obviously $\mathrm{P}(x, y)$ is $\Delta_{1}^{1}$ and cofinally progressive. By Theorem 3.4, there is a $\Pi_{1}^{1}$ set $A$ as described in the uniformization principle. One verifies that $A$ is an antichain of size $\aleph_{1}$.

It should be pointed out that a number of results concerning $\Pi_{1}^{1}$-ness- for example those considered in 13 - may be derived from Theorem 3.4. On the other hand, the existence of $\Pi_{1}^{1}$-maximal chains and antichains does not extend to all notions of reducibility. For example, there is no $\Pi_{1}^{1}$ set of reals whose hyperdegrees form a maximal chain. To see this, suppose that $A$ is such a set. Then $A$ does not contain a perfect subset since otherwise there will be two paths having incomparable hyperdegrees. By Theorem 2.3 of Mansfield and Solovay, this implies that $A \subset \mathcal{C}$. Let $\alpha$ be countable admissible that is also a limit of an increasing sequence of admissible ordinals $\left\{\alpha_{n}^{x_{n}} \mid n<\omega\right\}$ with $x_{n} \in A$. Then there is a real $x$ such that $\omega_{1}^{x}=\alpha$ [14. By the choice of $\alpha, x \notin \mathcal{C}$ and therefore $x \notin A$. However, $x_{n}<_{h} x$ for all $n$ and $x$ may be chosen to be in $L_{\omega_{1}^{y}}$ for any $y$ in $\mathcal{C}$ that is an upper bound (in the hyperdegrees) of $\left\{x_{n}\right\}$. Then $x<_{h} y$ for any such $y$. Since $A$ is maximal, this is a contradiction.

Observe also that there is no thin $\Pi_{1}^{1}$ set of reals whose hyperdegrees form an antichain. Indeed, if $A$ is such a set, then as above $A \subset \mathcal{C}$. But then $A$ is not an antichain.

\section{MARTIN'S CONJECTURES AND I}

In this section, we apply Theorem 3.4 to study Martin's conjectures under the assumption $V=L$. We begin by recalling some definitions.

Let $f: 2^{\omega} \rightarrow 2^{\omega}$.

Definition 5.1. (1) $f$ is degree invariant if and only if $\forall x \forall y\left(x \equiv_{\mathrm{T}} y \Longrightarrow\right.$ $\left.f(x) \equiv_{\mathrm{T}} f(y)\right)$.

(2) $f$ is uniformly degree invariant if and only if there is a function $t: \omega \rightarrow \omega$ such that for all $i, j<\omega$ and reals $x$ and $y, x=\Phi_{i}^{y} \wedge y=\Phi_{j}^{x} \Longrightarrow f(x)=$ $\Phi_{(t(\langle i, j\rangle))_{0}}^{f(y)} \wedge f(y)=\Phi_{\left(t(\langle i, j\rangle)_{1}\right.}^{f(x)}$.

(3) $f$ is increasing on a cone if and only if there is a real $y$ such that for all $x \geq_{\mathrm{T}} y, x \leq_{\mathrm{T}} f(x)$.

(4) $f$ is order preserving on a cone if and only if there is a real $y$ such that for all $x, z \geq_{\mathrm{T}} y, x \leq_{\mathrm{T}} z \Longrightarrow f(x) \leq_{\mathrm{T}} f(z)$.

(5) $f$ is constant on a cone if and only if there are reals $y$ and $y_{0}$ such that for all $x \geq_{T} y, f(x) \equiv_{T} y_{0}$.

Given degree-invariant functions $f$ and $g$, write $f \geq_{M} g$ if $f(x) \geq_{\mathrm{T}} g(x)$ on a cone. Martin's conjectures state that assuming $Z F$ together with $A D$ and the Axiom of Dependent Choice $(D C)$,

(I) Every degree-invariant function $f$ that is not increasing on a cone is constant on a cone; and

(II) $<_{M}$ is a pre-well-ordering on degree-invariant functions which are increasing on a cone. If $f$ has $<_{M}$-rank $\alpha$ in the pre-well-ordering, then $f^{\prime}$ has $<_{M^{-}}$ rank $\alpha+1$, where $f^{\prime}(x)=(f(x))^{\prime}$ by definition (' denotes Turing jump). 
Many results related to Martin's conjectures can be found in [19] and [16]. In general, since Borel determinacy is a theorem of $Z F+D C$ [12, Steel and SlamanSteel's results remain true for all $\boldsymbol{\Delta}_{1}^{1}$ (and hence $\boldsymbol{\Sigma}_{1}^{1}$ ) functions. Steel [19] proved that (II) is true if $f$ is, in addition, a uniformly degree-invariant and increasing function on a cone. Note that (II) implies that if $f$ is degree invariant and $f(x) \equiv_{\mathrm{T}} x$ cofinally (i.e., for every $z$, there is a real $x \geq_{\mathrm{T}} z$ such that $f(x) \equiv_{\mathrm{T}} x$ ), then $f(x) \equiv_{\mathrm{T}} x$ on a cone. We prove that this fails at the $\Pi_{1}^{1}$ level under the assumption $V=L$.

Theorem 5.2. 2 Assume $V=L$. There is a $\Pi_{1}^{1}$ uniformly degree-invariant function $g$ that is increasing and order preserving on a cone such that for all $y$, there are two reals $x_{0}, x_{1} \geq_{T}$ y satisfying $g\left(x_{0}\right) \equiv_{\mathrm{T}} x_{0}$ and $g\left(x_{1}\right) \geq_{\mathrm{T}} \mathcal{O}^{x_{1}}$.

Proof. Assume $V=L$. Define $\mathrm{P}(x, y)$ as

$$
\begin{aligned}
y \in L_{\omega_{1}^{y}} \wedge \mathcal{O}^{\mathcal{O}^{x}}=(y)_{0} \wedge(y)_{1}=x \wedge & (y)_{2} \\
& \text { is the }<_{L} \text {-least real so that }(y)_{2} \not \mathbb{Z}_{\mathrm{T}}(y)_{0} .
\end{aligned}
$$

Since " $\mathcal{O}^{\mathcal{O}^{x}}=(y)_{0}$ " is $\Pi_{1}^{1}$ (see [15]), $\mathrm{P}$ is a $\Pi_{1}^{1}$ cofinally progressive relation. By Theorem 3.4, there is a $\Pi_{1}^{1}$ set $A$ satisfying the prescribed properties in $\mathfrak{I}$.

By the definition of $\mathrm{P}$, every real is Turing below some real in $A$. Moreover, $\mathrm{P}$ ensures that $<_{\mathrm{T}}$ is a well-ordering and consistent with $<_{L}$ on $A$.

Define $f(x)=y$ if

(a) $y \in A$;

(b) $x \leq_{\mathrm{T}} y$;

(c) $x>_{\mathrm{T}} \emptyset \Longrightarrow \forall n\left(x \not \mathbb{\mathrm { T }}_{\mathrm{T}}\left((y)_{1}\right)_{n}\right)$.

Roughly speaking, $y$ is the $<_{\mathrm{T}}$-least real in $A$ such that $x \leq_{\mathrm{T}} y$.

By (c) and the property of $A, f$ is well-defined. Furthermore, $f$ is a $\Pi_{1}^{1}$-increasing, order preserving, and uniformly degree-invariant function $\left(x \equiv_{\mathrm{T}} y\right.$ implies $f(x)=$ $f(y))$. Moreover, since $f(x)=x$ for every $x \in A$, we have $f(x) \equiv_{\mathrm{T}} x$ cofinally.

For every real $s$, take a real $x_{s} \in A$ with $x_{s} \geq_{\mathrm{T}} s$. Then for the $<_{\mathrm{T}}$-least real $y \in A$ above $x_{s}$, there is a real $z$ coding $\left\{x \mid x<_{L} y \wedge x \in A\right\}=\left\{x \mid x<_{\mathrm{T}} y \wedge x \in A\right\}$ so that $\mathrm{P}(z, y)$ holds. Obviously $x_{s}^{\prime} \leq_{\mathrm{T}} z^{\prime} \leq_{\mathrm{T}} \mathcal{O}^{z}$ (where $x_{s}^{\prime}$ is the Turing jump of $\left.x_{s}\right)$. By the definition of $\mathrm{P}$,

$$
\mathcal{O}^{x_{s}^{\prime}} \leq_{\mathrm{T}} \mathcal{O}^{\mathcal{O}^{z}} \leq_{\mathrm{T}} y
$$

and $(y)_{1}=z$. Thus

$$
\left\{\left((y)_{1}\right)_{n} \mid n \in \omega\right\}=\left\{r \mid r \leq_{\mathrm{T}} x \wedge r \in A\right\} .
$$

Since $x_{s}^{\prime} \not_{\mathrm{T}} r$ for all $r \in A$ with $r \leq_{\mathrm{T}} x_{s}$, we have $x_{s}^{\prime} \not_{\mathrm{T}}\left((y)_{1}\right)_{n}$ for all $n \in \omega$. Hence $f\left(x_{s}^{\prime}\right)=y$.

In other words, $f\left(x_{s}^{\prime}\right) \geq_{\mathrm{T}} \mathcal{O}^{x_{s}^{\prime}}$.

Slaman and Steel [16] proved that assuming $A D$, if $f$ is uniformly degreeinvariant and not increasing on a cone, then $f$ is constant on a cone. We show that this also fails for $\Pi_{1}^{1}$ functions under the Axiom of Constructibility.

\footnotetext{
${ }^{2}$ Also proved independently by Slaman using a different argument.
} 
Proposition 5.3. Assume $V=L$. There is a uniformly degree-invariant, nonincreasing $\Pi_{1}^{1}$ function $f$ such that $\left.f(x)\right|_{\mathrm{T}} x$ (i.e. $f(x)$ and $x$ have incomparable Turing degrees) on a cone. In particular, $f$ is not constant on a cone.

Proof. Let $A$ be the $\Pi_{1}^{1}$ set as constructed in Theorem 5.2, Define $f(x)=y$ if and only if there is a real $z \equiv_{\mathrm{T}} y^{\prime}$ so that

(a) $z \in A$;

(b) $y$ has a minimal degree;

(c) $y=\Phi_{e}^{z}$ for some $e$ so that for all $i<e$, if $\Phi_{i}^{z}$ has a minimal degree, then $\left(\Phi_{i}^{z}\right)^{\prime} \neq_{\mathrm{T}} z ;$ and

(d) $x>_{\mathrm{T}} \emptyset \Longrightarrow \forall n\left(x \not \mathbb{\mathrm { T }}_{\mathrm{T}}\left((z)_{1}\right)_{n}\right)$.

We leave it to the reader to verify that $f$ is the desired counterexample.

As a final application of $\mathfrak{I}$, we show that (II) of Martin's conjectures fails at the $\Pi_{1}^{1}$ level in a strong way; i.e., there is a $<_{M}$-decreasing sequence, again under the assumption $V=L$.

Theorem 5.4. Assume $V=L$. There is a sequence of uniformly degree-invariant, increasing, $\Pi_{1}^{1}$ functions $\left\{f_{n}\right\}_{n}$ such that $f_{n+1}<_{M} f_{n}$ for all $n \in \omega$. Thus $<_{M}$ is not a pre-well-ordering.

Proof. Assume $V=L$. Define $\mathrm{P}(x, y)$ by

$$
\begin{aligned}
y \in L_{\omega_{1}^{y}} \wedge(y)_{0}=x \wedge(y)_{1} & \text { is }<_{L} \text {-least } \\
& \text { such that }(y)_{1} \not_{\mathrm{T}}(y)_{0} \wedge y \equiv_{\mathrm{T}}\left((y)_{0} \oplus(y)_{1} \oplus(y)_{2}\right)^{\prime} .
\end{aligned}
$$

Obviously $\mathrm{P}(x, y)$ is a $\Pi_{1}^{1}$ cofinally progressive relation. By Theorem 3.4 , there is a $\Pi_{1}^{1}$ set with the prescribed properties.

By the definition of $\mathrm{P}$, every real is Turing below some real in $A$. Moreover, by the definition of $\mathrm{P},<_{T}$ is a well-ordering and consistent with $<_{L}$ on $A$.

It is not difficult to see that there is an arithmetical set $W=\{(m, n, z) \mid m, n \in$ $\left.\omega \wedge z \in 2^{\omega}\right\}$ such that for each real $z,\left\{W_{n}^{z}\right\}_{n \in \omega}=\{\{m \mid(m, n, z) \in W\}\}_{n \in \omega}$ is a sequence of $z$-r.e. sets so that for all $n, z<_{\mathrm{T}} W_{n+1}^{z}<_{\mathrm{T}} W_{n}^{z}$.

Define a function $f_{n}(x)=y$ if there exists a real $z<_{\mathrm{T}} y$ such that

(a) $z \in A$;

(b) $z \geq_{\mathrm{T}} x$;

(c) $y=W_{n+1}^{z}$;

(d) $x>_{\mathrm{T}} \emptyset \Longrightarrow \forall m\left(x \not \underline{\mathrm{T}}_{\mathrm{T}}\left((z)_{0}\right)_{m}\right)$.

Roughly speaking, $y$ is the $W_{n+1}^{z}$ for the $<_{\mathrm{T}}$-least real $z$ in $A$ with $z \geq_{\mathrm{T}} x$.

Obviously $\left\{f_{n}\right\}_{n}$ is a sequence of $\Pi_{1}^{1}$ functions. Note that for any $x<_{\mathrm{T}} y$ in $A$, $x^{\prime} \leq_{\mathrm{T}} y$. So for every $n, f_{n}$ is degree invariant and increasing.

By (d) and the property of $A, f$ is well-defined.

By the choice of $\left\{W_{n}^{z}\right\}_{n \in \omega},\left\{f_{n}\right\}_{n \in \omega}$ is a $<_{M}$-descending sequence.

We end this paper with an open question:

Results in this section for $\Pi_{1}^{1}$ uniformly degree-invariant functions were proved under $V=L$. This means that Slaman-Steel's results in 16 may not hold even for $\Pi_{1}^{1}$ functions assuming a hypothesis different from $A D$ (or $\Pi_{1}^{1}$-determinacy). One may then wish to identify the consistency strength of (I) and (II) in Martin's 
conjectures. Thus, is the consistency of (I) and (II) (for uniformly degree-invariant functions, say) in the $\Pi_{1}^{1}$ case equivalent to a large cardinal hypothesis? Since $\Pi_{1}^{1}$-determinacy, which implies the Slaman-Steel results, is equiconsistent with the existence of $0^{\sharp}[9$, and the latter is stronger than a simple assumption of the existence of an inaccessible cardinal, the consistency strength of (I) and (II) for $\Pi_{1}^{1}$ uniformly degree-invariant functions becomes particularly interesting.

\section{ACKNOWLEDGMENTS}

The authors wish to thank the referee for bringing their attention to the work of Kenneth Kunen and Arnold Miller that preceded the current work. They are also grateful to the referee for the careful reading of the manuscript, as well as for suggestions that led to an improvement of the presentation of this paper.

\section{REFERENCES}

[1] Peter Aczel and Wayne Richter. Inductive definitions and analogues of large cardinals. In Conference in Mathematical Logic-London 1970 (Proc. Conf., Bedford Coll., London, 1970), pages 1-9. Lecture Notes in Math., Vol. 255. Springer, Berlin, 1972. MR0363841 (51:96)

[2] Jon Barwise. Admissible sets and structures. Springer-Verlag, Berlin, 1975. MR0424560 $(54: 12519)$

[3] George Boolos and Hilary Putnam. Degrees of unsolvability of constructible sets of integers. J. Symbolic Logic, 33:497-513, 1968. MR0239977 (39:1331)

[4] Douglas Cenzer. Analytic inductive definitions. J. Symbolic Logic, 39:310-312, 1974. MR0357089 (50:9557)

[5] C. T. Chong and Liang Yu. Maximal chains in the Turing degrees. J. Symbolic Logic, 72:12191227, 2007. MR2371202

[6] C. T. Chong and Liang Yu. Thin maximal antichains in the Turing degrees. In S. Barry Cooper, Benedikt Löwe, and Andrea Sorbi, editors, CiE, volume 4497 of Lecture Notes in Computer Science, pages 162-168. Springer, 2007.

[7] S. B. Cooper. Minimal degrees and the jump operator. J. Symbolic Logic, 38:249-271, 1973. MR0347572(50:75)

[8] Keith J. Devlin. Constructibility. Perspectives in Mathematical Logic. Springer-Verlag, Berlin, 1984. MR:750828 (85k:03001)

[9] Leo Harrington. Analytic determinacy and 0\#. J. Symbolic Logic, 43(4):685-693, 1978. MR.518675 (80b:03065)

[10] R. Björn Jensen. The fine structure of the constructible hierarchy. Ann. Math. Logic, 4:229308; erratum, ibid. 4 (1972), 443, 1972. MR0309729 (46:8834)

[11] Richard Mansfield. Perfect subsets of definable sets of real numbers. Pacific J. Math., 35:451457, 1970. MR0280380(43:6100)

[12] Donald A. Martin. Borel determinacy. Ann. of Math. (2), 102(2):363-371, 1975. MR0403976 (53:7785)

[13] Arnold W. Miller. Infinite combinatorics and definability. Ann. Pure Appl. Logic, 41(2):179203, 1989. MR983001 (90b:03070)

[14] Gerald E. Sacks. Countable admissible ordinals and hyperdegrees. Advances in Math., 20(2):213-262, 1976. MR0429523 (55:2536)

[15] Gerald E. Sacks. Higher recursion theory. Perspectives in Mathematical Logic. SpringerVerlag, Berlin, 1990. MR.1080970 (92a:03062)

[16] Theodore A. Slaman and John R. Steel. Definable functions on degrees. In Cabal Seminar 8185, volume 1333 of Lecture Notes in Math., pages 37-55. Springer, Berlin, 1988. MR960895 (89m:03033)

[17] Robert M. Solovay. On the cardinality of $\Sigma_{2}^{1}$ sets of reals. In Foundations of Mathematics (Symposium Commemorating Kurt Gödel, Columbus, Ohio, 1966), pages 58-73. Springer, New York, 1969. MR0277382(43:3115) 
[18] Clifford Spector. Recursive well-orderings. J. Symbolic Logic, 20:151-163, 1955. MR0074347 $(17: 570 \mathrm{~b})$

[19] John R. Steel. A classification of jump operators. J. Symbolic Logic, 47(2):347-358, 1982. MR654792(84i:03085)

Department of Mathematics, Faculty of Science, National University of Singapore, Lower Kent Ridge RoAd, Singapore 117543

E-mail address: chongct@math.nus.eud.sg

Institute of Mathematical Sciences, Nanjing University, Nanjing, Jiangsu Province 210093, People's Republic of China

E-mail address: yuliang.nju@gmail.com 